\title{
SUMMATION OF INFINITE SERIES
}

\author{
H. L. MANOCHA and B. L. SHARMA
}

(Received 13 January 1966)

Abstract. In this paper we obtain the sum of some infinite series involving hypergeometric functions of one or more variables.

\section{Introduction}

In this paper an attempt has been made to seek the aid of fractional derivatives, which has enabled us not only to prove the results already obtained by Carlitz [2] and Halim and Salam [5] but also to generalise quite a few of them.

For the sake of convenience the rules for fractional integration and differentiation are given below. Following Erdelyi [3] we write the rules governing fractional integration by parts in the form

$$
\int_{a}^{b} U \frac{d^{\lambda} V}{d(b-x)^{\lambda}} d x=\int_{a}^{b} V \frac{d^{\lambda} U}{d(x-a)^{\lambda}} d x
$$

The fractional derivatives occurring in (1) can be defined by integrals, if the real part of $\lambda$ is negative.

Thus

$$
\left.\begin{array}{l}
\frac{d^{\lambda} U}{d(x-a)^{\lambda}}=\frac{1}{\Gamma(-\lambda)} \int_{a}^{x}(x-y)^{-\lambda-1} U(y) d y \\
\frac{d^{\lambda} V}{d(b-x)^{\lambda}}=\frac{1}{\Gamma(-\lambda)} \int_{x}^{b}(y-x)^{-\lambda-1} V(y) d y
\end{array}\right\} R(\lambda)<0 .
$$

If $U$ and $V$ are expressible by means of the series of the types

$$
U=\sum A_{r}(x-a)^{\rho+r-1}, \quad V=\sum B_{s}(b-x)^{\delta+\imath-1},
$$

then the fractional derivatives are obtainable by differentiating these series term by term and using the definition

$$
\frac{d^{\lambda} \omega^{\mu-1}}{d \omega^{\lambda}}=\frac{\Gamma(\mu)}{\Gamma(\mu-\lambda)} \omega^{\mu-\lambda-1}
$$

which holds for all values of $\lambda$ except $\lambda=\mu$.

We denote (4) by the operator notation as 
5)

$$
D_{\omega, \mu-1}^{\lambda}[\quad]=\frac{\Gamma(\mu)}{\Gamma(\mu-\lambda)}[]
$$

there $D_{\omega, \mu-1}^{\lambda}\left[\omega^{\alpha}\right]$ will stand for $\frac{d^{\lambda}\left[\omega^{\alpha+\mu-1}\right]}{d \omega^{\lambda}}$.

We give below a few elementary results which will be used in our inestigation.

6) $D_{x, \mu-1}^{\mu-\lambda}\left[(1-x)^{-\alpha}\right]=\frac{\Gamma(\mu)}{\Gamma(\lambda)} x^{\lambda-1} F_{1}(\alpha, \mu ; \lambda ; x)$

7) $D_{x, \mu-1}^{\mu-\lambda}\left[(1-x y)^{-\alpha}(1-x z)^{-\beta}\right]=\frac{\Gamma(\mu)}{\Gamma(\lambda)} x^{\lambda-1} F_{1}(\mu ; \alpha, \beta ; \lambda ; x y, x z)$

8) $D_{x, \mu-1}^{\mu-\lambda}\left[e^{x \nu}(1-x z)^{-\beta}\right]=\frac{\Gamma(\mu)}{\Gamma(\lambda)} x^{\lambda-1} \phi_{1}(\mu ; \beta ; \lambda ; x y, x z)$

9) $D_{x, \mu-1}^{\mu-\lambda}\left[e^{x y}(1-x z)^{-\alpha}(1-x U)^{-\beta}\right]=\frac{\Gamma(\mu) x^{\lambda-1}}{\Gamma(\lambda)} \phi_{1}(\mu ; \alpha, \beta ; \lambda ; x y, x z, x U)$.

ior the definitions of hypergeometric series of two or more variables, ee [1].

In our investigation, we require the definition of Laguerre polynomial, lainville $[6$, p. 200] as follows

$$
L_{n}^{(\alpha)}(x)=\frac{(1+\alpha)_{n}}{n !}{ }_{1} F_{1}(-n ; 1+\alpha ; x) .
$$

10) can be expressed in the following forms:

$$
L_{n}^{(\alpha)}(x)=\frac{(-x)^{n}}{n !}{ }_{2} F_{0}\left(-n,-\alpha-n ;-;-\frac{1}{x}\right)
$$

r

12)

$$
L_{n}^{(\alpha-n)}(x)=\frac{(-x)^{n}}{n !}{ }_{2} F_{0}\left(-n,-\alpha ;-;-\frac{1}{x}\right) .
$$

\section{2}

Let us start with the elementary result

$$
e^{(1-x) t}=e^{t} e^{-x t}
$$

Iultiplying both the sides by $(1-U x)^{m}$ and employing the operator $\underset{x, \alpha-1}{\alpha-\gamma}$, we get 


$$
\sum_{n=0}^{\infty} \frac{1}{n !} F_{1}(\alpha ;-n,-m ; \gamma ; x, U x) t^{n}=e^{t} \phi_{1}(\alpha ;-m ; \gamma ;-x t, U x) .
$$

Assuming $U=1$ and using the formula [4, p. 239]

$$
F_{1}\left(\alpha ; \beta, \beta^{\prime} ; \gamma ; x, x\right)={ }_{2} F_{1}\left(\alpha, \beta+\beta^{\prime} ; \gamma ; x\right),
$$

in (13), it gives

$$
\sum_{n=0}^{\infty} \frac{t^{n}}{n !}{ }_{2} F_{1}(-m-n, \alpha ; \gamma ; x)=e^{t} \phi_{1}(\alpha ;-m ; \gamma ;-x t, x) .
$$

If in (15) we replace $x$ by $x / \alpha$ and let $\alpha \rightarrow \infty$ then using (10), we get

$$
\sum_{n=0}^{\infty} \frac{(m+n) !}{n !(1+\gamma)_{m+n}} L_{m+n}^{(\gamma)}(x) t^{n}=e^{t} \phi_{3}(-m ; 1+\gamma ; x,-x t) .
$$

In case $m=0,(16)$ reduces to a well known generating function, Rainville $[6$, p. 201].

But if in (15) we multiply $x$ by $\gamma$ and let $\gamma \rightarrow \infty$, with the help of (12), we obtain

$$
\begin{aligned}
& \sum_{n=0}^{\infty} \frac{(m+n) !}{m ! n !} x^{m+n} L_{m+n}^{(-\alpha-m-n)}\left(-\frac{1}{x}\right) t^{n} \\
& =e^{t}(1+x t)^{-\alpha}\left(\frac{x}{1+x t}\right)^{m} L_{m}^{(-\alpha-m)}\left(-\frac{1+x t}{x}\right)
\end{aligned}
$$

which on changing $x, t$ and $\alpha$ into $-1 / x,-x t$ and $-\alpha-m$ respectively, it becomes the formula due to Carlitz [2]

$$
\sum_{n=0}^{\infty}\left(\begin{array}{c}
m+n \\
m
\end{array}\right) L_{m+n}^{(\alpha-n)}(x) t^{n}=(1+t)^{\alpha} e^{-x t} L_{m}^{\alpha}[x(1+t)]
$$

Returning to (13), we replace $t$ by $(1-y) t$, multiply both the sides by $(1-v y)^{k}$ and apply the operator $D_{\gamma, \boldsymbol{\beta}-1}^{\beta-\delta}$ thus getting

$$
\begin{aligned}
\sum_{n=0}^{\infty} \frac{t^{n}}{n !} F_{1}(\alpha ;-n,-m ; \gamma ; x, U x) F_{1}(\beta ;-n,-k ; \delta ; y, v y) \\
=e^{t} \sum_{n=0}^{\infty} \frac{(\alpha)_{n}(\beta)_{n}}{(\gamma)_{n}(\delta)_{n}} \frac{(x y t)^{n}}{n !} \phi_{1}(\alpha+n ;-m ; \gamma+n ;-x t, U x) \\
\phi_{1}(\beta+n ;-k ; \delta+n ;-y t, v y) .
\end{aligned}
$$

On putting $U=v=1$ and using (14), (18) becomes 


$$
\begin{array}{r}
\sum_{n=0}^{\infty} \frac{t^{n}}{n !}{ }_{2} F_{1}(-m-n, \alpha ; \gamma ; x)_{2} F_{1}(-k-n, \beta ; \delta ; y) \\
=e^{t} \sum_{n=0}^{\infty} \frac{(\alpha)_{n}(\beta)_{n}}{(\gamma)_{n}(\delta)_{n}} \frac{(x y t)^{n}}{n !} \phi_{1}(\alpha+n ;-m ; \gamma+n ;-x t, x) \\
\phi_{1}(\beta+n ;-k ; \delta+n ;-y t, y)
\end{array}
$$

which on putting $m=k=0$ further reduces to

$$
\begin{array}{r}
\sum_{n=0}^{\infty} \frac{t^{n}}{n !}{ }_{2} F_{1}(-n, \alpha ; \gamma ; x)_{2} F_{1}(-n, \beta ; \delta ; y) \\
=e^{t} \sum_{n=0}^{\infty} \frac{(\alpha)_{n}(\beta)_{n}}{(\gamma)_{n}(\delta)_{n}} \frac{(x y t)^{n}}{n !}{ }_{1} F_{1}(\alpha+n ; \gamma+n ;-x t) \\
{ }_{1} F_{1}(\beta+n ; \delta+n ;-y t) .
\end{array}
$$

Now if we put $x=1, y=1$ and $\delta=\gamma-\alpha$ then with the aid of Kummer's transformation $[4$, p. 253]

$$
{ }_{1} F_{1}(a ; c ; x)=e^{x}{ }_{1} F_{1}(c-a ; c ;-x),
$$

(20) yields the most interesting result of the paper

$$
\begin{aligned}
{ }_{1} F_{1}(\alpha+\beta ; \gamma ; t)=\sum_{n=0}^{\infty} \frac{(\alpha)_{n}(\beta)_{n}}{(\gamma)_{n}(\gamma-\alpha)_{n}} \frac{(-t)^{n}}{n !}{ }_{1} F_{1}(\alpha+n ; \gamma+n ; t) \\
{ }_{1} F_{1}(\beta+n ; \gamma-\alpha+n ; t) .
\end{aligned}
$$

Further on putting $\alpha=-m, \beta=-k$ and changing $\gamma$ into $\gamma+1$ in (21) and using the result (10), we obtain a known result due to Carlitz [2]

$$
\left(\begin{array}{c}
m+k \\
m
\end{array}\right) L_{m+k}^{(\gamma)}(t)=\sum_{n=0}^{\min (m \cdot k)} \frac{(-t)^{n}}{n !} L_{k-n}^{(y+m+n)}(t) L_{(m-n)}^{(y+n)}(t) .
$$

Turning now. to (19), if we multiply $x$ and $y$ by $y$ and $\delta$ respectively and let both $\gamma \rightarrow \infty$ and $\delta \rightarrow \infty$ and using (11) we get after a little simplification

$$
\begin{aligned}
\sum_{n=0}^{\infty} \frac{t^{n}}{n !}\left(\begin{array}{c}
m+n \\
m
\end{array}\right)\left(\begin{array}{c}
k+n \\
k
\end{array}\right) L_{m+n}^{(\alpha-n)}(x) L_{k+n}^{(\beta-n)}(y) \\
=e^{x y t}(1-x t)^{\beta}(1-y t)^{\alpha} \sum_{n=0}^{\infty} \frac{(-\alpha-m)_{n}(-\beta-k)_{n}}{n !} \\
\times\left[\frac{t}{(1-x t)(1-y t)}\right]^{n} L_{m}^{(\alpha-n)}[x(1-y t)] L_{k}^{(\beta-n)}[y(1-x t)] .
\end{aligned}
$$

On putting $m=0, k=0,(23)$ yields a known result due to Carlitz [2]. 


$$
\begin{aligned}
& \sum_{n=0}^{\infty} \frac{t^{n}}{n !} L_{n}^{(\alpha-n)}(x) L_{n}^{(\beta-n)}(y) t^{n} \\
& \quad=\beta ! e^{x y t}(1-y t)^{\alpha-\beta} t^{\beta} L_{\beta}^{(\alpha-\beta)}\left[-\frac{(1-x t)(1-y t)}{t}\right] .
\end{aligned}
$$

Returning to (18), we take $m=0$ and replace $x$ by $x y$ then taking $\gamma \rightarrow \infty$, we obtain with the help of (12) and (11)

$$
\begin{aligned}
& \sum_{n=0}^{\infty} L_{n}^{(\alpha-n)}(x) F_{1}(\beta ;-n,-k ; \delta ; y, v y) t^{n} \\
& =\left((1+t)^{\alpha} e^{-x t} \phi_{1}\left(\beta ;-\alpha,-k ; \delta ; x y t, \frac{y t}{1+t}, v y\right),\right.
\end{aligned}
$$

and

$$
\begin{aligned}
\sum_{n=0}^{\infty} \frac{t^{n}}{n !} L_{\alpha}^{(n-\alpha)}(x) F_{1}(\beta ;-n,-k ; \delta ; y, v y) t^{n} \\
\quad=\frac{(t-x)^{\alpha} e^{t}}{\alpha !} \phi_{1}\left(\beta ;-\alpha,-k ; \delta ;-y t, \frac{y t}{t-x}, v y\right) .
\end{aligned}
$$

(25) and (26) reduce to known results due to Halim and Salam [5], in case we take $k=0$.

\section{4}

In this section, we consider the well known generating function of the Laguarre polynomials

$$
\sum_{n=0}^{\infty} L_{n}^{(\alpha-n)}(x) t^{n}=(1-t)^{\alpha} e^{-x t}
$$

Changing $t$ into $u t$, multiplying both the sides by $(1-u y)^{m}$ and then employing the operator $D_{u, \beta-1}^{\beta-\gamma}$, we get

$$
\begin{array}{r}
\sum_{n=0}^{\infty} \frac{(\beta)_{n}}{(\gamma)_{n}} L_{n}^{(\alpha-n)}(x)_{2} F_{1}(-m, \beta+n ; \gamma+n ; y) t^{n} \\
=\phi_{1}(\beta ;-\alpha,-m ; \gamma ;-x t,-t, y) .
\end{array}
$$

Replacing $t$ and $y$ by $t / \beta$ and $y / \beta$ respectively and taking $\beta \rightarrow \infty$, we get

$$
\begin{aligned}
\sum_{n=0}^{\infty} \frac{m !}{(1+\gamma)_{m+n}} & L_{n}^{(\alpha-n)}(x) L_{m}^{(\gamma+n)}(y) t^{n} \\
& =\phi_{3}(-\alpha,-m ; 1+\gamma ;-x t,-t, y) .
\end{aligned}
$$


In the last, we consider

$$
\begin{aligned}
& e^{x(1-y) t} e^{-y(1-x) t}=e^{x t} e^{-y t} \\
& \quad \sum_{m, n=0}^{\infty} \frac{x^{m}(-y)^{n}(1-y)^{m}(1-x)^{n} t^{m+n}}{m ! n !}=\sum_{m, n=0}^{\infty} \frac{(x)^{m}(-y)^{n} t^{m+n}}{m ! n !} .
\end{aligned}
$$

Applying the operator $D_{x, \lambda-1}^{\lambda-\alpha}$ and $D_{y, \mu-1}^{\mu-\beta}$ on both sides, we have after a little simplification

$$
\begin{aligned}
\sum_{n=0}^{\infty} & \sum_{m=0}^{n} \frac{(\lambda)_{m}(\mu)_{n-m}}{m !(n-m) !(\alpha)_{m}(\beta)_{n-m}} x^{m}(-y)^{n-m} t^{n} \\
& { }_{2} F_{1}[-(n-m), \lambda+m ; \alpha+m ; x]_{2} F_{1}[-m, \mu+n-m ; \beta+n-m ; y] \\
\quad= & \sum_{n=0}^{\infty}{ }_{3} F_{2}\left[-n, \lambda, 1-\beta-n ; \alpha, 1-\mu-n ; \frac{x}{y}\right] t^{n} .
\end{aligned}
$$

On comparing the coefficients of $t^{n}$ on both sides, we obtain

$$
\begin{aligned}
& \sum_{m=0}^{n}\left(\begin{array}{l}
n \\
m
\end{array}\right) \frac{(\lambda)_{m}(1-\beta-n)_{m}}{(\alpha)_{m}(1-\mu-n)_{m}}\left(-\frac{x}{y}\right)^{m}{ }_{2} F_{1}[-(n-m), \lambda+m ; \alpha+m ; x] \\
&{ }_{2} F_{1}[-m, \mu+n-m ; \beta+n-m ; y] \\
&={ }_{3} F_{2}\left[\left[-n, \lambda, 1-\beta-n ; \alpha, 1-\mu-n ; \frac{x}{y}\right] .\right.
\end{aligned}
$$

We shall obtain some interesting particular cases of (31).

(i) By putting $x=y=1$, we obtain

(32) ${ }_{3} F_{2}\left[\begin{array}{c}-n, \lambda, \beta-\mu ; 1 \\ 1+\lambda-\alpha-n, 1-\mu-n\end{array}\right]=\frac{(\lambda)_{n}}{(\alpha-\lambda)_{n}} F_{2}\left[\begin{array}{c}-n, \lambda, 1-\beta-n ; 1 \\ \alpha, 1-\mu-n\end{array}\right]$.

(ii) Dividing $x$ and $y$ by $\lambda$ and $\mu$ respectively and taking $\lambda \rightarrow \infty$, $\mu \rightarrow \infty$, changing $\alpha$ and $\beta$ into $1+\alpha$ and $1+\beta$ respectively and using (10), we get

$$
\begin{aligned}
& \sum_{m=0}^{n} \frac{(-\beta-n)_{m}}{(1+\alpha)_{m}} L_{m}^{(\alpha)}(x) L_{n \rightarrow m}^{(\beta)}(y) \\
& \quad=\frac{(-1)^{n}(x+y)^{n}}{(1+\alpha)_{n}} P_{n}^{(\alpha, \beta)}\left(\frac{y-x}{y+x}\right) .
\end{aligned}
$$

In particular for $y=-x$,

$$
\begin{gathered}
\sum_{m=0}^{n} \frac{(-\beta-n)_{m}}{(1+\alpha)_{m}} L_{m}^{(\alpha)}(x) L_{n-m}^{(\beta)}(-x) \\
=\frac{(x)^{n}(1+\alpha+\beta)_{2 n}}{(1+\alpha)_{n}(1+\beta)_{n}(1+\alpha+\beta)_{n}} .
\end{gathered}
$$




\section{References}

[1] P. Appell and J. Kampé de Feriet, Fonctions hypergéometriques et hypersphériques, polynomes d'Hermite, Gauthier-Villars, Paris (1926).

[2] L. Carlitz, 'A note on the Laguerre polynomials', Michigan Mathematical Journal. 7 (1960), 219-223.

[3] A. Erdélyi, 'On fractional integration and also its application to the theory of Hankel transform', Quart. J. Math. (Oxford) 11 (1940), 292-303.

[4] A. Erdélyi, Higher transcendental functions, Vol I (1953).

[5] N. A. Halim and W. A. Al-Salam, 'Double Euler transformations of certain hypergeometric functions', Duke Jour. Maths. 30 (1963), $51-62$.

[6] E. D. Rainville, Special functions, New York (1960).

Department of Applied Sciences

Punjab Engineering College

Chandigarh, India 